\title{
RESEARCH HIGHLIGHT Resident macrophages keep mitochondria running in the heart
}

\author{
Ronald J. Vagnozzi ${ }^{1,2}$ and Jeffery D. Molkentin ${ }^{1,3}$ \\ Cell Research (2020) 30:1057-1058; https://doi.org/10.1038/s41422-020-00427-z
}

\begin{abstract}
The high workload of cardiac myocytes, coupled with their negligible turnover, requires that compensatory mechanisms act in the heart to address ongoing cellular damage and extruded organelle components that would otherwise accumulate. A recent study in Cell by Nicolás-Ávila et al. illustrates how cardiac-resident macrophages serve such a role, by clearing extruded mitochondrial debris to preserve organ homeostasis and maintain optimal cardiac function.
\end{abstract}

Cardiac myocytes are long-lived, terminally differentiated cells specialized for contraction. In addition to being comprised largely of myofilament proteins, these cells possess an abundant and complex mitochondrial network that generates sufficient energetic capacity to maintain cardiac output. ${ }^{1}$ Given their extremely long life-span, cardiac myocytes rely heavily on systems for proteostasis and organelle surveillance and clearance, such as ERassociated degradation (ERAD), proteosome-mediated quality control, autophagy and mitophagy, that collectively preserve myocyte integrity. ${ }^{2}$ These mechanisms are primarily myocyteautonomous but a growing body of literature implicates substantial regulatory contribution from non-myocytes in the heart, particularly fibroblasts, immune cells, and vascular cells. ${ }^{3}$ However, the extent to which each interstitial cell population contributes directly or indirectly to overall cardiac myocyte homeostasis remains undefined.

Macrophages comprise the majority of immune interstitial cells in the heart under steady state, ${ }^{4}$ and recent studies have used sophisticated genetic fate mapping and immunophenotyping to clarify the diverse nature of these cells at baseline or upon tissue damage. ${ }^{5-7}$ Tissue-resident macrophages arise from the fetal yolk sac and fetal liver during development and seed distal organs, including the heart. This population of macrophages is augmented and progressively replaced by circulating monocyte-derived macrophages throughout postnatal life or after tissue injury. ${ }^{5}$ Beyond variations in ontogeny, cardiac macrophages (cMacs) are remarkably plastic and can acquire differential gene expression or context-dependent cellular phenotypes, based on local tissue cues. Much of the focus regarding CMac biology has centered around inflammatory responses to tissue damage (such as myocardial infarction) or pathogens, but more current work has uncovered novel and unexpected physiological roles of cMacs at steady state. ${ }^{8}$ For example, cardiac-resident macrophages are involved in propagation of electrical current at the atrioventricular (AV) node. ${ }^{9}$ Depletion of macrophages in otherwise healthy mice led to cardiac conduction defects, specifically AV block, suggesting a key role for resident macrophages in regulating cardiac pacemaker activity.

Publishing recently in Cell, the Hidalgo and Enríquez laboratories have added to our growing understanding of physiological roles for cardiac tissue macrophages. Nicolás-Ávila et al. demonstrated that cMacs actively phagocytose mitochondrial fragments released from myocytes, thus maintaining both myocyte and interstitial homeostasis. ${ }^{10}$ The authors employed optical tissue clearing and genetic ablation to functionally characterize the $\mathrm{cMac}$ pool at steady state. Using CX3CR1-GFP knock-in mice to fluorescently label cMacs, along with CD169-driven expression of diphtheria toxin receptor (DTR) for inducible macrophage ablation, they observed an intricate network of cMacs in close proximity to each myocyte (Fig. 1a). Depletion of CD169expressing CMacs triggered progressive cardiomyopathy within three weeks, with reduced cardiac output, impaired relaxation (diastolic dysfunction), and compromised hemodynamics. These findings implicate tissue macrophages as unanticipated regulators of overall cardiac function. Interestingly, cMac-depleted hearts eventually recovered from functional impairment after an additional three weeks. Given previously reported rates of cMac turnover and proliferation, ${ }^{5,8}$ this suggests that the authors observed a transient cardiomyopathy that could be reversed by intrinsic restoration of the cMac population. Alternatively, distinct populations of macrophages arising from circulating monocytes could be compensating for the loss of cMacs in this model, ${ }^{6}$ particularly given the potential for inflammatory cytokine production in the setting of cMac ablation. To address this, future studies to define cumulative dynamics of immune cells (and other nonmyocytes) in the context of genetic macrophage depletion will be needed.

To determine potential mechanisms underlying the effects of cMac depletion, Nicolás-Ávila et al. performed proteomic profiling of cMac-depleted hearts, noting an altered mitochondrial proteome suggestive of mitochondrial impairment. Further analysis of these hearts revealed accumulation of defective mitochondria which displayed ultrastructural defects in vivo and reduced ATP generation ex vivo. In a series of elegant genetic experiments using fluorescently labeled cardiac myocytes or mitochondrial DNA, the authors demonstrated mitochondrial material originating from myocytes within cMacs in vivo, indicating that cMacs actively phagocytose extracellular mitochondrial fragments. Of note, this process occurred at least partly via the myocyte autophagy machinery, culminating in release of LC3containing membrane vesicles that were engulfed by cMacs (Fig. 1a). Nicolás-Ávila et al. termed these extruded components as exophers, based on similarity to previously reported vesicles in $C$. elegans neurons.

By modulating autophagic flux using either rapamycin or myocyte-specific Atg7-hemizygous mice, the authors showed that myocyte autophagy is a central driver of exopher release. Improper clearance of exophers in cMac-depleted hearts was also

\footnotetext{
${ }^{1}$ Department of Pediatrics, University of Cincinnati, Cincinnati Children's Hospital Medical Center, Cincinnati, OH, USA; ${ }^{2}$ Division of Cardiology, Department of Medicine,

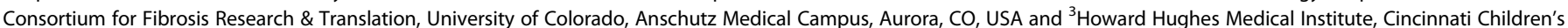
Hospital Medical Center, Cincinnati, OH, USA

Correspondence: Ronald J. Vagnozzi (ronald.vagnozzi@cuanschutz.edu) or Jeffery D. Molkentin (jeff.molkentin@cchmc.org)
}

Published online: 2 November 2020 


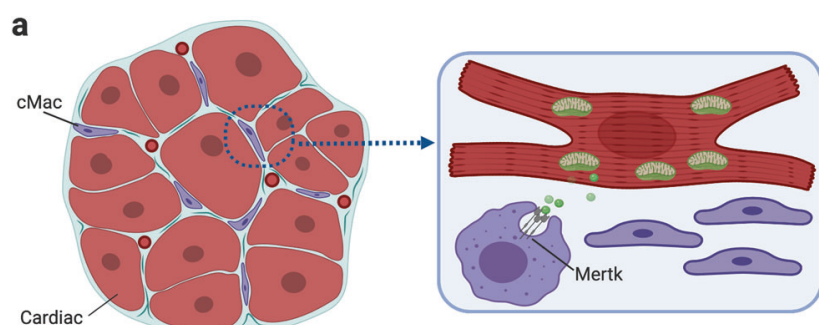

Myocyte

b

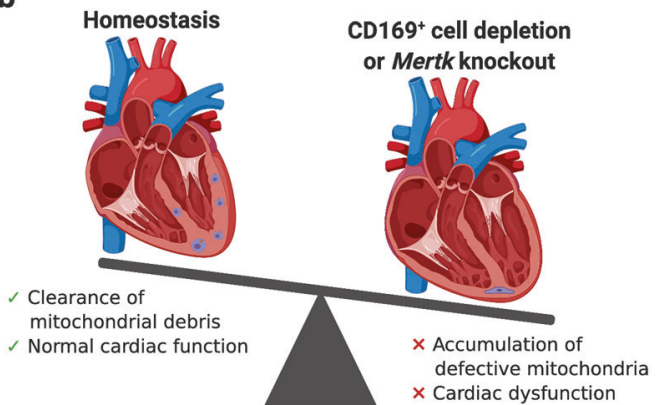

Fig. 1 Cardiac-resident macrophages maintain cellular and functional homeostasis in the heart. a Cardiac tissue macrophages (cMacs: $\mathrm{CD}_{4}{ }^{+} \mathrm{CD} 11 \mathrm{~b}^{+} \mathrm{F} 4 / 80^{+}$and marked by CX3CR1-GFP) reside in the interstitial compartment of the heart at baseline, with multiple cMacs surrounding each cardiac myocyte. cMacs actively phagocytose mitochondrial debris released from cardiac myocytes under physiological conditions, via the receptor tyrosine kinase Mertk. b Genetic ablation of cMacs using CD169-driven DTR, or knockout of Mertk, triggers accumulation of mitochondrial debris, impaired autophagy, and NLRP3 inflammasome activation, resulting in compromised heart function and metabolic derangement. Illustration was created with BioRender.com.

associated with inflammatory stress, as indicated by elevated NLRP3 inflammasome levels and caspase-1 cleavage. Moreover, adrenergic stress or infarction injury potentiated release of mitochondrial fragments in the heart, while depletion of cMacs worsened cardiac dysfunction in these models. Finally, NicolásÁvila et al. demonstrated that the phagocytosis receptor Mertk is a key downstream effector for uptake of mitochondrial material by cMacs. Mertk-deficient hearts recapitulated several aspects of cMac depletion, including accumulation of damaged and energetically compromised mitochondria, metabolic impairment, inflam- masome activation, and diastolic dysfunction (Fig. 1b). These data provide further evidence supporting a key role for macrophage activity, specifically phagocytosis, in maintaining cardiac homeostasis. Interestingly, prior data indicate differential phagocytic efficiency among cMac subsets. ${ }^{5}$ Whether uptake of exophers or mitochondria-derived material differs between embryonic- and monocyte-derived macrophages in the heart, and how this may impact tissue maintenance in homeostatic versus injury contexts, awaits further exploration.

Overall, the recent work of Nicolás-Ávila et al. demonstrates yet another crucial function of tissue macrophages in the heart, and highlights how immune cells can impact diverse physiological processes beyond inflammation and immunity. The mechanism of exopher-mediated communication proposed by Nicolás-Ávila et al. also raises several unanswered questions, including how this process is regulated at the level of myocytes and the mitochondrial network specifically (perhaps through mitophagy or mitochondrial fusion/fission dynamics), or how both myocytes and macrophages are able to sense the presence or absence of exophers in the interstitium and respond accordingly. How macrophages might secondarily impact overall intracellular myocyte proteostasis ${ }^{2}$ or mitochondrial function ${ }^{1}$ was not addressed here, but this question will be important for future investigation.

Nicolás-Ávila et al. underscores broader lingering questions in the field, including precisely how macrophages or other interstitial cells regulate cardiac myocytes in vivo within the tissue microenvironment. Specifically, it is still unclear how reciprocal signals between interstitial macrophages and myocytes (via exophers or otherwise) would traverse across the basal lamina to influence cell or organ function in a regulated fashion. Nevertheless, the highly plastic nature and multifaceted origins of tissue macrophages suggest that even more unexpected features of this dynamic cell type will emerge in the future.

\section{REFERENCES}

1. Murphy, E. et al. Circ. Res. 118, 1960-1991 (2016).

2. Henning, R. H. \& Brundel, B. Nat. Rev. Cardiol. 14, 637-653 (2017).

3. Forte, E., Furtado, M. B. \& Rosenthal, N. Nat. Rev. Cardiol. 15, 601-616 (2018).

4. Pinto, A. R. et al. Circ. Res. 118, 400-409 (2016).

5. Epelman, S. et al. Immunity 40, 91-104 (2014).

6. Bajpai, G. et al. Circ. Res. 124, 263-278 (2019).

7. Chakarov, S. et al. Science 363, eaau0964 (2019).

8. Ma, Y., Mouton, A. J. \& Lindsey, M. L. Transl. Res. 191, 15-28 (2018).

9. Hulsmans, M. et al. Cell 169, 510-522 (2017).

10. Nicolas-Avila, J. A. et al. Cell 183, 94-109 (2020). 